\title{
EQUILIBRIUM SHAPE OF PRISMATIC DISLOCATION LOOPS UNDER UNIFORM STRESS
}

\author{
R. B. CALHOUN and A. MORTENSEN†t \\ Massachusetts Institute of Technology, Cambridge, MA 02139, U.S.A.
}

(Received 10 November 1998; accepted 14 April 1999)

\begin{abstract}
The rolling, recrystallization and cooling of $\mathrm{AgCl}$ containing $1-5 \mu \mathrm{m}$ glass spheres generates thermal misfit dislocations. Under stress, prismatic loops elongate in the glide cylinder defined by their line sense and Burgers vector. Using optical microscopy, the shape of dislocation loops under an applied stress of $2.3 \mathrm{MPa}$ is measured. The measurements are corrected for a friction stress of $0.34 \mathrm{MPa}$ and compared with a model which incorporates the orientation dependent line tension (ODLT) of a dislocation. The measured data show considerable scatter; after averaging, good agreement between theory and experiment is obtained. (C) 1999 Acta Metallurgica Inc. Published by Elsevier Science Ltd. All rights reserved.
\end{abstract}

Keywords: Composites; Dislocations; Compounds, ionic; Optical microscopy; Microstructure

\section{INTRODUCTION}

Prismatic dislocation loops are closed dislocations which, in an unstressed crystal, lie in the plane normal to their Burgers vector $\vec{b}$. As a shear stress is applied, a prismatic loop elongates in the glide cylinder defined by $\vec{b}$ and the dislocation's line sense $\hat{\xi}$. Once $\hat{\xi}$ is parallel to $\vec{b}$, the dislocation can cross slip and escape the glide cylinder. Nabarro [1] discusses the development of this concept; it is illustrated and described qualitatively by Read [2].

The equilibrium shape of a loop under stress and the critical shear stress $\tau^{*}$ required for cross slip were calculated by Grilhé [3] under the assumption of constant dislocation line tension. Grilhé found that $\tau^{*}=W_{\mathrm{s}} / b r$, where $r$ is the radius and $W_{\mathrm{s}}$ the energy per unit length of dislocation. Grilhé also predicted that supercritical loops and helices should form Frank-Read sources.

In the present study we use a decoration technique to measure loop shape as a function of the applied stress in $\mathrm{AgCl}$. The data compare favorably with an extension of Grilhés model which incorporates a constant matrix friction stress term and the orientation dependent line tension (ODLT) of a dislocation as it changes in character from edge to screw.

\section{THEORY}

\subsection{Orientation dependent line tension solution}

In what follows we assume the matrix is isotropic and the stress state uniform, that dynamic effects

$\dagger$ Present address: Department of Materials, Swiss Federal Institute of Technology in Lausanne, CH-1015 Lausanne, Switzerland.

$\$$ To whom all correspondence should be addressed. can be ignored, and, because the effects of nonadjacent dislocation components are small for large $(r \gg b)$ loops [4], that the "line tension" approximation can be used. (Limitations of the line tension concept are discussed in Chapter 6 of Hirth and Lothe [5].)

Consider an initially circular prismatic dislocation loop in a glide cylinder of radius $r$ and axis coincident with the $\hat{e}_{3}^{\text {loop }}$ basis vector of the orthonormal system $\hat{e}_{i}^{\text {loop }}$. Angle $\theta$ sweeps the plane normal to $\hat{e}_{3}^{\text {loop }}$ starting at $\hat{e}_{1}^{\text {loop }}$, Fig. 1. Then loop $\vec{s}$ is described by

$$
\vec{s}(\theta)=\left[\begin{array}{c}
r \cos \theta \\
r \sin \theta \\
r A(\theta)
\end{array}\right]
$$

where $A(\theta)$ is its normalized elongation. The Burgers vector is $(0,0, b)$. Using the $F S / R H$ convention [5], $b$ is negative for an interstitial loop.

The force due to the uniform stress tensor $\sigma^{\text {loop }}$ is computed using the Peach-Koehler equation [6]. After defining a resolved shear stress term $\tau=$ $\sigma_{13} \sec \alpha$ where $\tan \alpha=-\sigma_{23} / \sigma_{13}$ and rewriting the result in the orthonormal basis

$$
\hat{t}=\left[\begin{array}{c}
-\sin \theta \\
\cos \theta \\
0
\end{array}\right], \hat{z}=\left[\begin{array}{l}
0 \\
0 \\
1
\end{array}\right], \hat{r}=\left[\begin{array}{c}
\cos \theta \\
\sin \theta \\
0
\end{array}\right]
$$

we obtain the glide $(\hat{t}$ and $\hat{z})$ and climb $(\hat{r})$ components of the unit force $\vec{F}_{\text {s }}$

$$
\vec{F}_{\mathrm{s}}=-\frac{b}{\sqrt{1+A^{\prime}(\theta)^{2}}}\left[\begin{array}{c}
A^{\prime}(\theta) \tau \cos (\alpha+\theta) \\
-\tau \cos (\alpha+\theta) \\
A^{\prime}(\theta) \tau \sin (\alpha+\theta)+\sigma_{33}
\end{array}\right] .
$$

Resisting elongation is the increase in the dislocation's self-energy. The energy per unit length of a straight dislocation is, using Foreman's [7] notation 


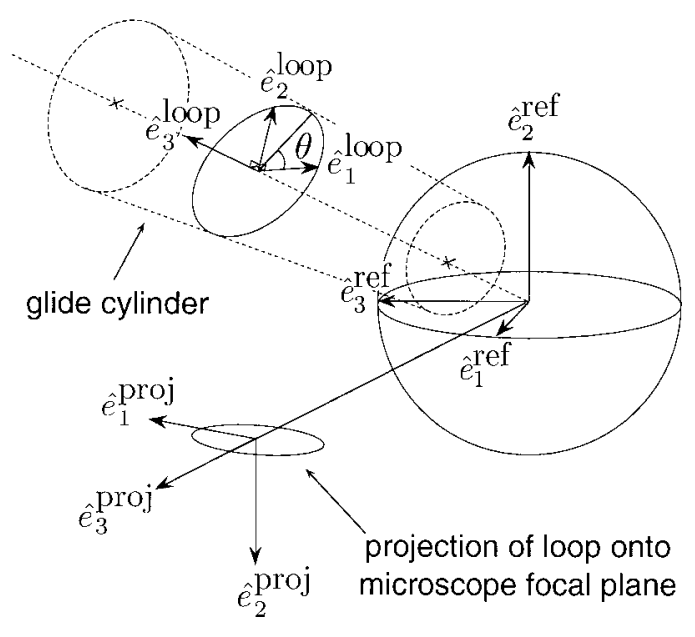

Fig. 1. Coordinate systems used in analyzing loop shape Basis $\hat{e}_{i}^{\text {loop }}$ is fixed with respect to each loop; $\hat{e}_{i .}^{\text {ref }}$ is fixed with respect to the applied stress direction; $\hat{e}_{i}^{\text {proj }}$ is based on a projection of $\hat{e}_{i}^{\text {loop }}$ onto the focal plane of the microscope.

$$
W_{\mathrm{s}}(\beta)=\frac{K(\beta) b^{2}}{4 \pi} \ln \left(\frac{R}{r_{0}}\right) \approx \frac{K(\beta) b^{2}}{4 \pi} \ln \left(\frac{r}{b}\right) .
$$

In an isotropic medium, the energy coefficient $K(\beta)$ is $G\left(\cos ^{2} \beta+\sin ^{2} \beta /(1-v)\right)$, where $\beta$ is the angle between $\vec{b}$ and $\hat{\xi}$ and $G$ is the elastic shear modulus.

The effective line tension of a curved dislocation [8] is $W_{\mathrm{s}}(\beta)+\partial^{2} W_{\mathrm{s}}(\beta) / \partial \beta^{2}$. The restoring force $\vec{F}_{\text {r }}$ is proportional to the line tension and to the local curvature $\kappa$

$$
\begin{aligned}
\vec{F}_{\mathrm{r}}= & -\frac{W_{\mathrm{s}}(0)\left((1+v) A^{\prime}(\theta)^{2}+1-2 v\right)}{r(1-v)\left(1+A^{\prime}(\theta)^{2}\right)^{3}} \\
& \cdot\left[\begin{array}{c}
A^{\prime}(\theta) A^{\prime \prime}(\theta) \\
-A^{\prime \prime}(\theta) \\
1+A^{\prime}(\theta)^{2}
\end{array}\right] .
\end{aligned}
$$

At equilibrium, $\vec{F}_{\mathrm{s}}+\vec{F}_{\mathrm{r}}=0$ for all $\theta$. Both $\hat{t}$ and $\hat{z}$ components yield, after integration, the differential equation

$$
\begin{aligned}
& -\Omega \sin (\alpha+\theta)+c \\
& =A^{\prime}(\theta) \frac{1-2 v+(1-v) A^{\prime}(\theta)^{2}}{(1-v)\left(1+A^{\prime}(\theta)^{2}\right)^{3 / 2}}
\end{aligned}
$$

where we have introduced the dimensionless stress parameter $\Omega=r b \tau / W_{\mathrm{s}}(0)$. As $A^{\prime}(\theta)$ must be periodic on the interval $0-2 \pi$ for a single prismatic loop, $c=0$.

Squaring this equation yields a cubic equation in $A^{\prime}(\theta)^{2}$, which was solved using Mathematica (Wolfram Research, Champaign, IL) to yield lengthy polynomials in $\Omega \sin (\alpha+\theta)$ and $v$. With $\sqrt{ }(F(\theta))$ the real root, continuity requirements dictate that

$$
A^{\prime}(\theta)=\left\{\begin{array}{ll}
-\sqrt{F(\theta)} & \text { when }-\alpha<\theta \leq \pi-\alpha \\
\sqrt{F(\theta)} & \text { when } \pi-\alpha<\theta \leq 2 \pi-\alpha
\end{array} .\right.
$$

$A(\theta)$ is obtained by numerical integration.

When $A^{\prime}(\theta) \longrightarrow \pm \infty$, the dislocation has screw character and can cross slip. Since $A^{\prime}(\pi / 2-\alpha)$ has denominator 0 when $\Omega=1$ and, for $0 \leq v \leq 1 / 2$, negative and non-zero numerator, $A^{\prime}( \pm \pi / 2-$ $\alpha) \longrightarrow \infty$ when $\Omega \longrightarrow \pm 1$. Therefore

$$
\Omega^{*}= \pm 1
$$

where $\Omega^{*}$ is the dimensionless stress above which equilibrium cannot be obtained.

\subsection{Grilhé's solution}

We recover Grilhé's solution by setting $W_{\mathrm{s}}(\beta)$ equal to the self-energy of a pure screw dislocation, $W_{\mathrm{s}}(0)$, and integrating twice. Since $A(\theta)=0$ at $\theta=$ $\pm \pi / 2-\alpha$

$$
\begin{aligned}
A(\theta)= & \ln \left(\Omega \cos (\alpha+\theta)+\sqrt{1-\Omega^{2} \sin ^{2}(\alpha+\theta)}\right) \\
& -\frac{1}{2} \ln \left(1-\Omega^{2}\right) .
\end{aligned}
$$

For this case, $A^{\prime}( \pm \pi / 2-\alpha) \longrightarrow \mp \infty$ as $\Omega \longrightarrow \pm 1$, so again $\Omega^{*}= \pm 1$.

\subsection{Effects of crystal anisotropy}

The ODLT analysis assumes an isotropic matrix, whereas crystals of $\mathrm{AgCl}$ and many metals of engineering interest display cubic symmetry. We applied Foreman's anisotropic analysis, as presented in Hirth and Lothe [5], to calculate the line tension $W_{\mathrm{s}}(\beta)+\partial^{2} W_{\mathrm{s}}(\beta) / \partial \beta^{2}$ for dislocations in $\mathrm{AgCl}$. Using the elastic constants of Loje and Schuele [9] $\left(c_{11}=59.85, c_{12}=36.11, c_{44}=6.24 \mathrm{GPa}\right.$ at $\left.300 \mathrm{~K}\right)$, we computed the effective line tension for dislocations with $\vec{b}=\frac{1}{2}[101]$ as a function of $\beta$, the angle between a pure [101] screw dislocation and various edge orientations. The results are plotted in Fig. 2. The curves for dislocations with edge components of [101] and [010] show the range of values obtained when using anisotropic elasticity. We computed the mean value of the anisotropic line tensions for all dislocations which make angle $\beta$ with [101] for comparison with the isotropic line tension and constant line tension cases. All curves are normalized by the line energy of a pure screw dislocation, $W_{\mathrm{s}}(0)$.

The anisotropic dislocation line tension depends on the orientation of a dislocation segment with respect to the crystallographic axes. Because the anisotropy of $\mathrm{AgCl}$ is relatively strong, the shape of a prismatic loop will depend both on its orientation with respect to the applied stress and on the crystal orientation. Computation of dislocation shape is difficult in the anisotropic case; however, Fig. 2 shows that the mean anisotropic line tension is reasonably well approximated by the isotropic expression. Thus, while anisotropy effects will cause 


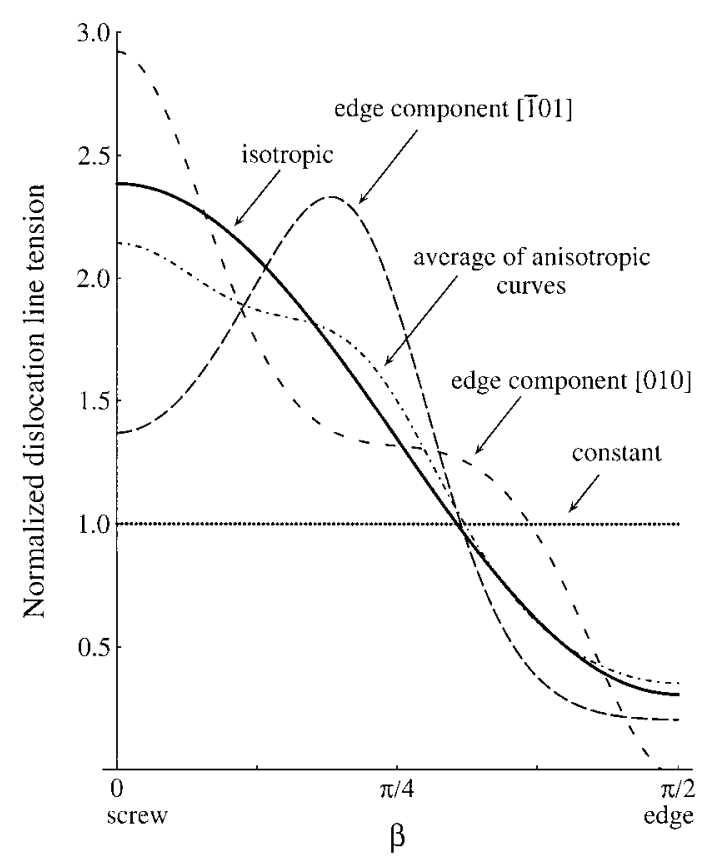

Fig. 2. Dislocation line tension $W_{\mathrm{s}}(\beta)+\partial^{2} W_{\mathrm{s}}(\beta) / \partial \beta^{2}$ for dislocations in $\mathrm{AgCl}$ with $\vec{b}=\frac{1}{2}[101]$ normalized by energy of a pure screw dislocation $W_{\mathrm{s}}(0)$. Anisotropic elasticity was used to compute the line tension of dislocations with edge components [101] and [010] and the average line tension for all dislocations at angle $\beta$ to $\vec{b}$. The curve computed using isotropic elasticity (solid line) is fairly close to the average of the anisotropic results and is used in the present model. Also shown is the line tension of a dislocation where the line energy is assumed to be constant.

the behavior of individual loops to deviate from the values predicted by the isotropic ODLT model, the mean response of many loops of different crystallographic orientations should approach the values predicted by the isotropic elasticity calculations.

\subsection{Incorporating a non-zero flow stress}

The ODLT model also ignores lattice friction. To a first approximation, the effects of the Peierls stress and interactions with solute atoms or second phase precipitates can be modeled with a constant friction stress term, $\tau_{\mathrm{f}}$. The relative importance of $\tau_{\mathrm{f}}$ and the dislocation's self-energy are compared in the dimensionless friction stress $v$ of Bullough and Newman [10] where $v=4 \pi r(1-v) \tau_{\mathrm{f}} / G b$.

When $v$ is greater than about 5 , dislocation behavior is dominated by matrix friction. When $0<v<5$, matrix friction can be accommodated in the ODLT model by incorporating $\tau_{\mathrm{f}}$ into $\Omega$ :

$$
\Omega=\left\{\begin{array}{ll}
\frac{\tau}{|\tau|} \cdot \frac{r b\left(|\tau|-\tau_{\mathrm{f}}\right)}{W_{\mathrm{s}}(0)} & \text { when }|\tau|-\tau_{\mathrm{f}} \geq 0 \\
0 & \text { when }|\tau|-\tau_{\mathrm{f}}<0
\end{array} .\right.
$$

This will compensate, on average, for interactions between a dislocation and randomly distributed obstacles.

\section{EXPERIMENT}

\subsection{Experimental procedures}

We compared the predictions of the ODLT model with experiment using $150 \mu \mathrm{m}$ thick samples of the ductile ionic solid $\mathrm{AgCl}$ containing 0.01 vol. $\%$ of $1-5 \mu \mathrm{m}$ pyrex microspheres. Samples were prepared by homogenizing an ingot of $\mathrm{AgCl}$ containing the microspheres and 500 p.p.m. $\mathrm{CuCl}$ sensitizer, rolling a piece of the ingot to the target thickness, cutting the sheet into tensile specimens and recrystallizing these specimens at $573 \mathrm{~K}$. Prismatic loops are generated in this material during cooling from the matrix recrystallization temperature due to the different thermal expansion properties of the two phases.

Freshly recrystallized samples of dimensions $37 \times$ $3 \times 0.15 \mathrm{~mm}^{3}$ were glued by their ends onto the top of a $13 \mathrm{~mm}$ thick beam. The glue was a catalyzed cyanoacrylate adhesive; $3 \mathrm{M}$ Scotchgard was used as a stop layer and lubricant. After waiting overnight for the glue to fully cure, the beam was loaded in three-point bending as shown in Fig. 3. This yields a sample stress state which is close to uniaxial tension. Beam deformation was monitored with strain gauges; sample stress was calculated from the applied beam strain and the stress-strain curves [11] of identically heat-treated $\mathrm{AgCl}$ from the same ingot.

Samples were illuminated with an unfiltered xenon bulb strobe lamp while under load. This catalyzes the decomposition of silver chloride into metallic silver, which subsequently precipitates on dislocations and other defects in the crystal. Dislocations are locked in their elongated state by the decoration process, preserving their shape for later examination with an optical microscope.

This technique [11], a modification of that developed by Mitchell and co-workers [12-15], produces dislocations that are resolvable in the top $40 \mu \mathrm{m}$ of a sample when oil-immersion optics are used. The examined volumes are much greater than in electron-transparent metal samples, and even large $(5 \mu \mathrm{m})$ loops are relatively free from the effects of free surfaces. The ability to fix dislocation configurations under load using only visible/near UV light is a significant advantage of dislocation decoration. Compared with the technique of Mitchell and coworkers, the present technique has the advantage of producing pore-free samples, such that uniform deformation produces a uniform stress distribution across the sample.

\subsection{Physical constants}

The Voigt average elastic constants of $\mathrm{AgCl}$ are $G=8.49 \mathrm{GPa}$ and $v=0.409$. From the X-ray diffraction data of Fouchaux and Simmons [16], $b=$ $0.392 \mathrm{~nm}$ at $300 \mathrm{~K}$.

The friction stress $\tau_{\mathrm{f}}$ was deduced from the spacing between prismatic dislocations generated by 


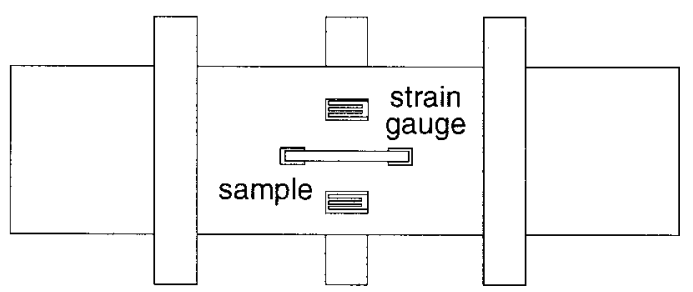

scale:
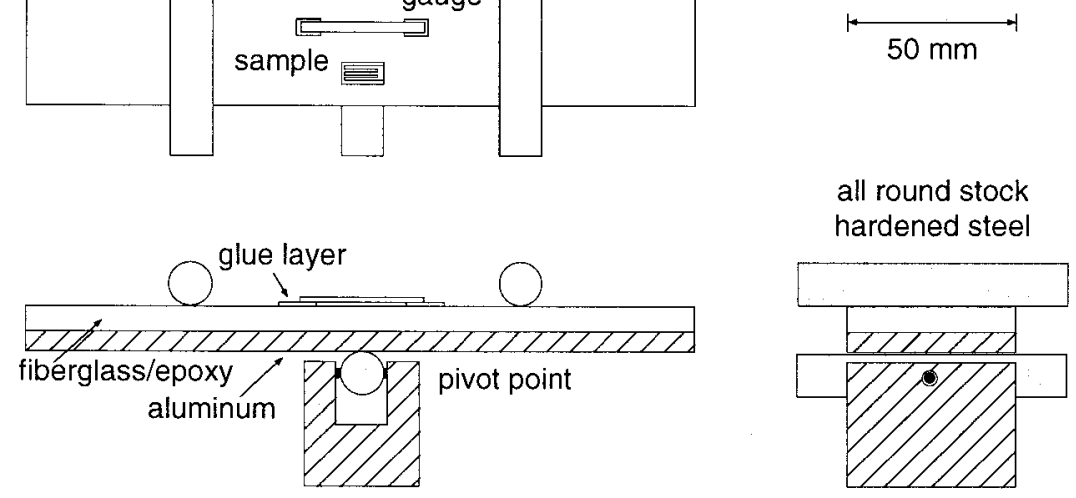

Fig. 3. Tensile testing rig used with decoration samples. Sample dimensions are approximately $37 \times 3 \times 0.15 \mathrm{~mm}^{3}$. Glued regions on each end are approximately $3 \times 3 \mathrm{~mm}^{2}$.

thermal mismatch. Bullough and Newman [10] analyzed the spacing between such prismatic dislocations, correlating the dimensionless loop spacing $\chi=x / 2 r$ with the dimensionless friction stress $v$. Dunand and Mortensen [17] used their results to determine the spacing $\chi_{0}$ between the penultimate and last loops, finding that these expressions could be approximated by the function $\log v_{0}=0.866-1.08 \chi_{0}+0.102 \chi_{0}^{2}$.

Eighteen values of $\chi_{0}$ were determined from an identically annealed $\mathrm{AgCl} /$ glass sample to which no external stress was applied. Loop spacings were measured using the image processing software IPLab (Scanalytics, Inc., Fairfax, VA) and corrected for the position of the observer. Loop diameters were taken to be the mean of the measured diameters of the two loops. The mean of the values of $\tau_{\mathrm{f}}$ computed was $0.34 \pm 0.06 \mathrm{MPa}$, so $v$ is in the range $0.4-2$.

\subsection{Data processing}

Only distinctly closed loops were used: figureeight loops, incompletely formed (shear) loops, and helical structures were ignored. To avoid complications caused by unrelaxed thermal stresses in the vicinity of glass spheres, data were gathered using only the outermost loop of each punched loop train. Loops less than four radii from the center of the glass sphere or from the surface of the sample were also excluded. We take the stress state in the vicinity of loops meeting these criteria to be one of simple tension.

In the sample coordinate system $\hat{e}_{i}^{\text {ref }}$, shown in Fig. 1, the tensile load is applied along $\hat{e}_{3}^{\text {ref }}$ and the microscope axis is parallel to $\hat{e}_{2}^{\text {ref }}$. We define angle $\omega$ to be the complement of the angle between $\hat{e}_{3}^{\text {ref }}$ and $\hat{e}_{3}^{\text {loop }}$, and angle $\phi$ to be the angle between $\hat{e}_{1}^{\text {ref }}$ and the projection of $\hat{e}_{1}^{\text {loop }}$ onto the plane normal to $\hat{e}_{3}^{\text {ref }}$ as shown in Fig. 4. In terms of the reference frame $\hat{e}_{i}^{\text {ref }}$, the basis vectors $\hat{e}_{i}^{\text {loop }}$ are

$$
\begin{gathered}
\hat{e}_{1}^{\text {loop }}=\left[\begin{array}{c}
\cos \phi \sin \omega \\
\sin \phi \sin \omega \\
-\cos \omega
\end{array}\right], \hat{e}_{2}^{\text {loop }}=\left[\begin{array}{c}
-\sin \phi \\
\cos \phi \\
0
\end{array}\right], \\
\hat{e}_{3}^{\text {loop }}=\left[\begin{array}{c}
\cos \phi \cos \omega \\
\sin \phi \cos \omega \\
\sin \omega
\end{array}\right] .
\end{gathered}
$$

Experimentally, $\phi$ and $\omega$ are computed from the crystallographic orientation of each grain and the fact that $\hat{e}_{3}^{\text {loop }}$ is a $\langle 110\rangle$ direction. Grain orientation is determined from the angle that each $\langle 110\rangle$ punching direction makes with $\hat{e}_{3}^{\text {ref }}$ when projected onto the plane normal to $\hat{e}_{2}^{\text {ref }}$. This process is described in Appendix A.

Rotation of the stress tensor into the loop frame yields $\tau=-\frac{1}{2} \sigma_{33} \sin 2 \omega$ and $\alpha=0$. Since $b$ is negative for interstitial loops, $\Omega$ is positive and the loop reaches its maximum extent $A^{\text {max }}$ at $A(0)$. We use $A^{\max }$ as a measure for comparing the predictions of the model with experiment.

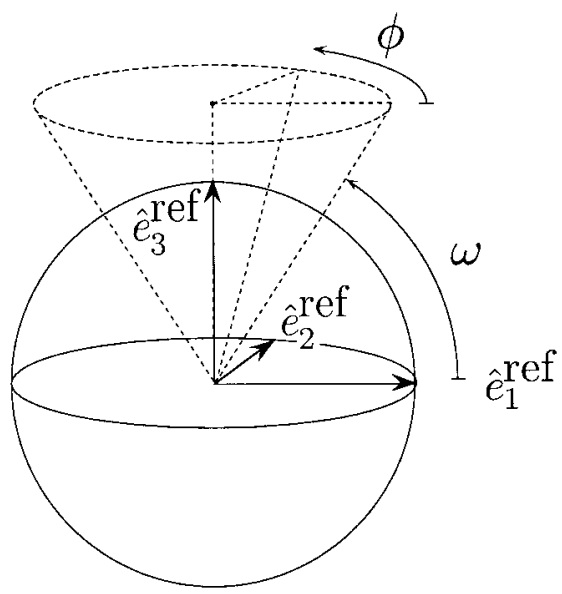

Fig. 4. Definition of angles used to describe a loop's orientation with respect to the loading direction $\hat{e}_{3}^{\text {ref }}$. 

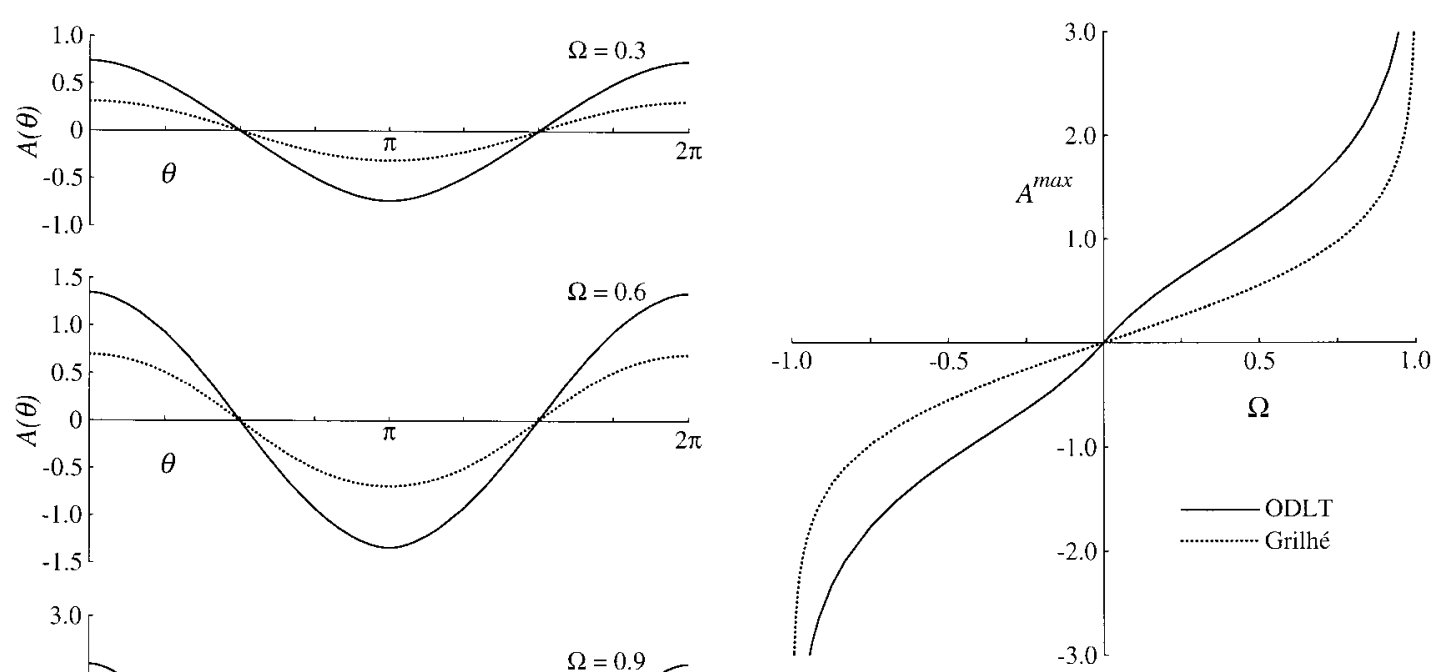

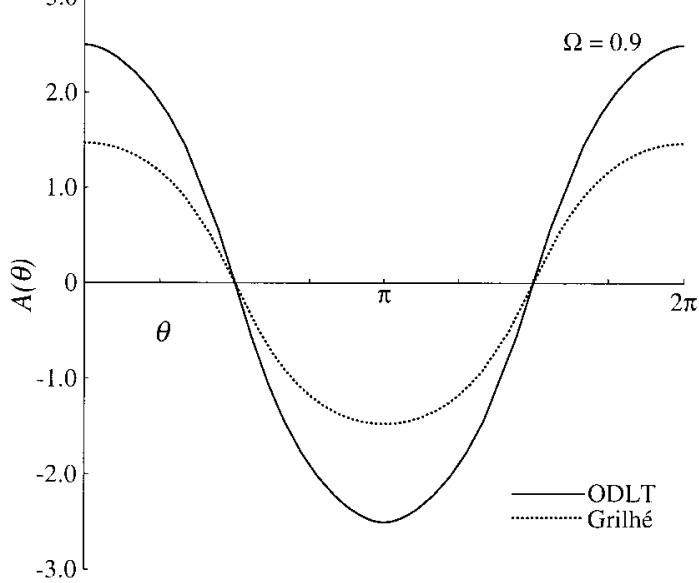

Fig. 5. Plots of $A(\theta)$ vs $\theta$ for ODLT and Grilhé models of loop shape for $\Omega=0.3,0.6,0.9$ where $\Omega$ is a dimensionless shear stress. Computation assumes isotropic elasticity with $v=0.409$.

Except for the special case where $\phi$ equals 0 or $\pi$, $A^{\max }$ has to be computed from an experimentally measurable quantity, the apparent canting angle. This process is described in the Appendix A. The uncertainties associated with the correction process increase as $\phi \longrightarrow n \pi / 2$, sometimes generating clearly erroneous results. These data were discarded.

\section{RESULTS AND DISCUSSION}

Predicted loop geometries under stress are plotted in their "unrolled" shape [plots of $A(\theta)$ vs $\theta$ ] in Fig. 5. The Grilhé and ODLT analyses predict identical critical values of $\Omega$ but differ in their predictions of loop shape at smaller values of $\Omega$. Note that the ODLT model predicts a longer and more canted loop at each $\Omega$ despite the fact that the dislocation line energy is everywhere equal to or higher than the constant term $W_{\mathrm{s}}(0)$ used in the Grilhe model. This is because the dislocation line tension contains a torque term which results in an effective line tension smaller than $W_{\mathrm{s}}(0)$ for dislocations
Fig. 6. Plot of $A^{\max }$ vs $\Omega$ for ODLT and Grilhé models of loop elongation for an isotropic matrix with $v=0.409$. At small values of $\Omega$, the ODLT analysis predicts a more canted loop. Both models predict the same critical value.

which are predominantly edge in character $(\cos \beta>1 / \sqrt{ } 3$. This is shown in Fig. 2 .

Plots of the predicted magnitude of canting $A^{\max }$ as a function of $\Omega$ are given in Fig. 6 for a crystal with Poisson ratio $v$ of 0.409 . A function which approximates the ODLT solution for $\Omega$ less than about 0.95 is

$$
\begin{aligned}
& A^{\max } \approx\left(1.12+10.5 v^{2}\right) \Omega-\left(0.593+16.8 v^{2}\right) \Omega^{3}+(1.85 \\
& \left.\quad+14.3 v^{2}\right) \Omega^{5} .
\end{aligned}
$$

An example of loops in their unstressed configuration is shown in Fig. 7. (Loops with Burgers vectors in the plane of focus look like straight line segments in micrographs.) Examples of the effect of stress on such loops can be seen in Figs 8 and 9 . The loading axis is left-right in these micrographs; loops under stress cant towards the loading axis and the magnitude of canting increases as the orientations of maximum shear are approached.

Quantitative measurements of loop canting were made on a sample loaded in tension to an applied strain of $191 \mu \epsilon$, which corresponds to a stress of 2.3 MPa. Values of $\Omega$ were determined for each measured loop using the definition of $\Omega$ given in equation (9) with $\tau_{\mathrm{f}}=0.34 \mathrm{MPa}$. As collected, the axis of symmetry of the canting data did not coincide with the applied stress direction: there was an angle of $0.143 \mathrm{rad}$ between the two. This could result from several causes, including uneven load transfer across the glued sample ends and crystal anisotropy. Assuming this discrepancy resulted primarily from a small local rotation of the principal stress axis in the sample under examination, we rotated the direction of the applied stress by $0.143 \mathrm{rad}$ when computing values of $\Omega$ and $A^{\max }$ so 


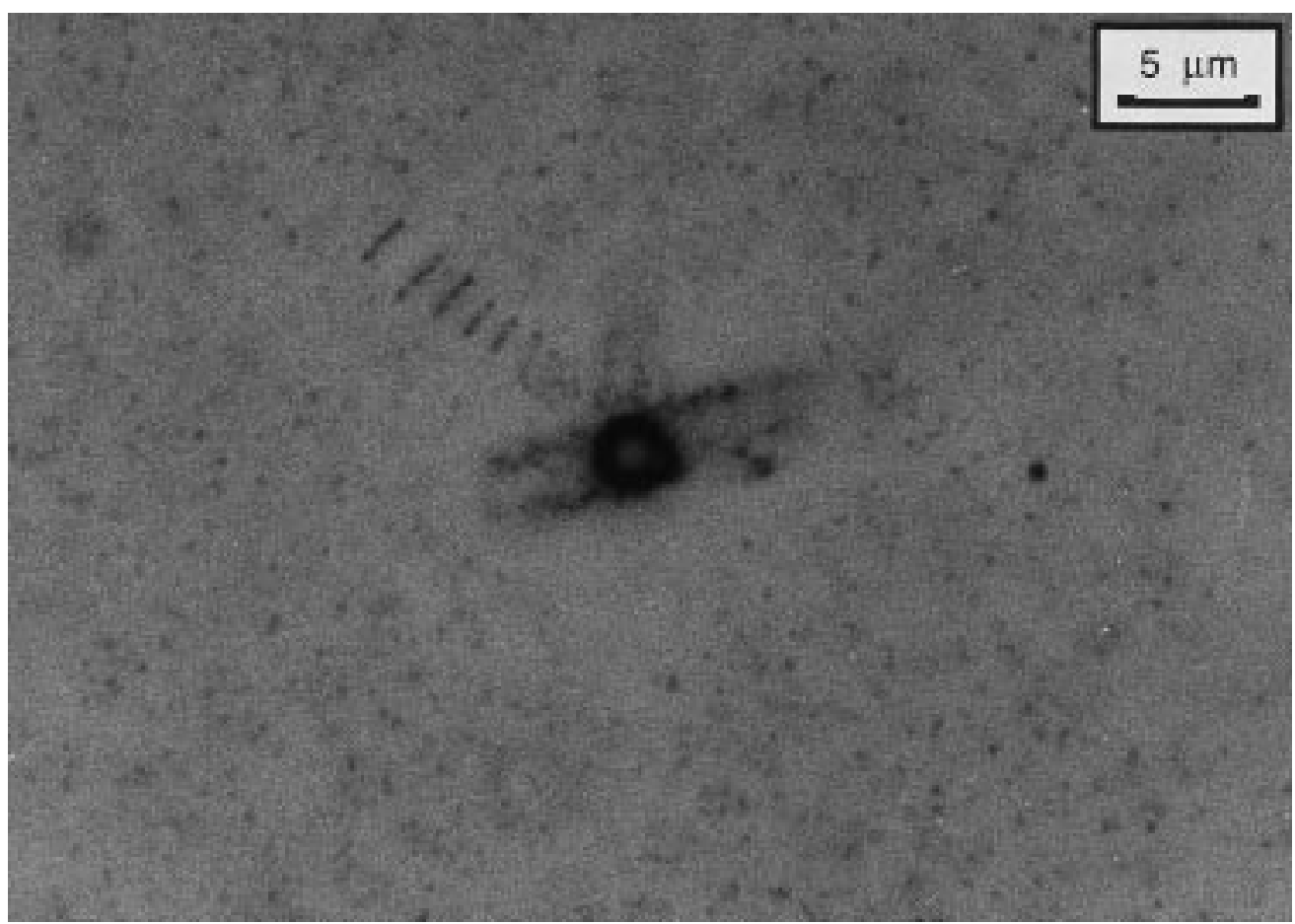

Fig. 7. Prismatic dislocation loops punched by glass spheres due to thermal mismatch. No external stress has been applied. Discrete loops with $\vec{b}$ in the plane of focus (above and left of sphere) resemble line segments. Depth of sphere in the crystal is approximately $22 \mu \mathrm{m}$.

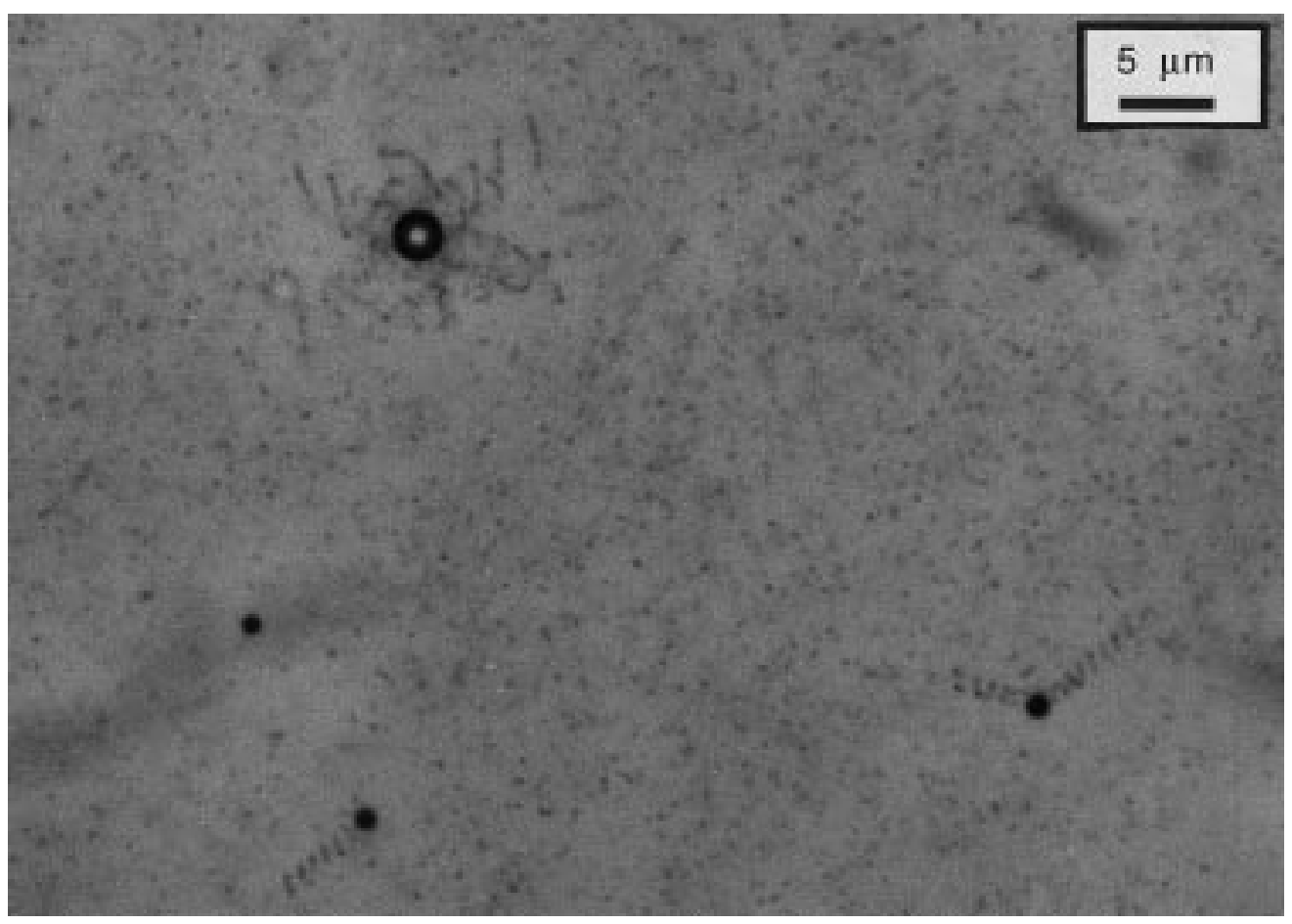

Fig. 8. Dislocation loops canting under an applied stress. Figure shows canting of dislocations generated by a glass sphere (top center) and by two smaller silver precipitates (lower left and lower right). Loading direction is left-right to a far-field stress of approximately $2.1 \mathrm{MPa}$. The shear stress varies as $\sin 2 \omega$, where $\omega$ is the angle which $\vec{b}$ makes with the loading direction. Prismatic loops cant towards the applied stress axis, with the magnitude of canting increasing with the resolved shear stress. The depth of the spheres in the crystal is approximately $20 \mu \mathrm{m}$. 


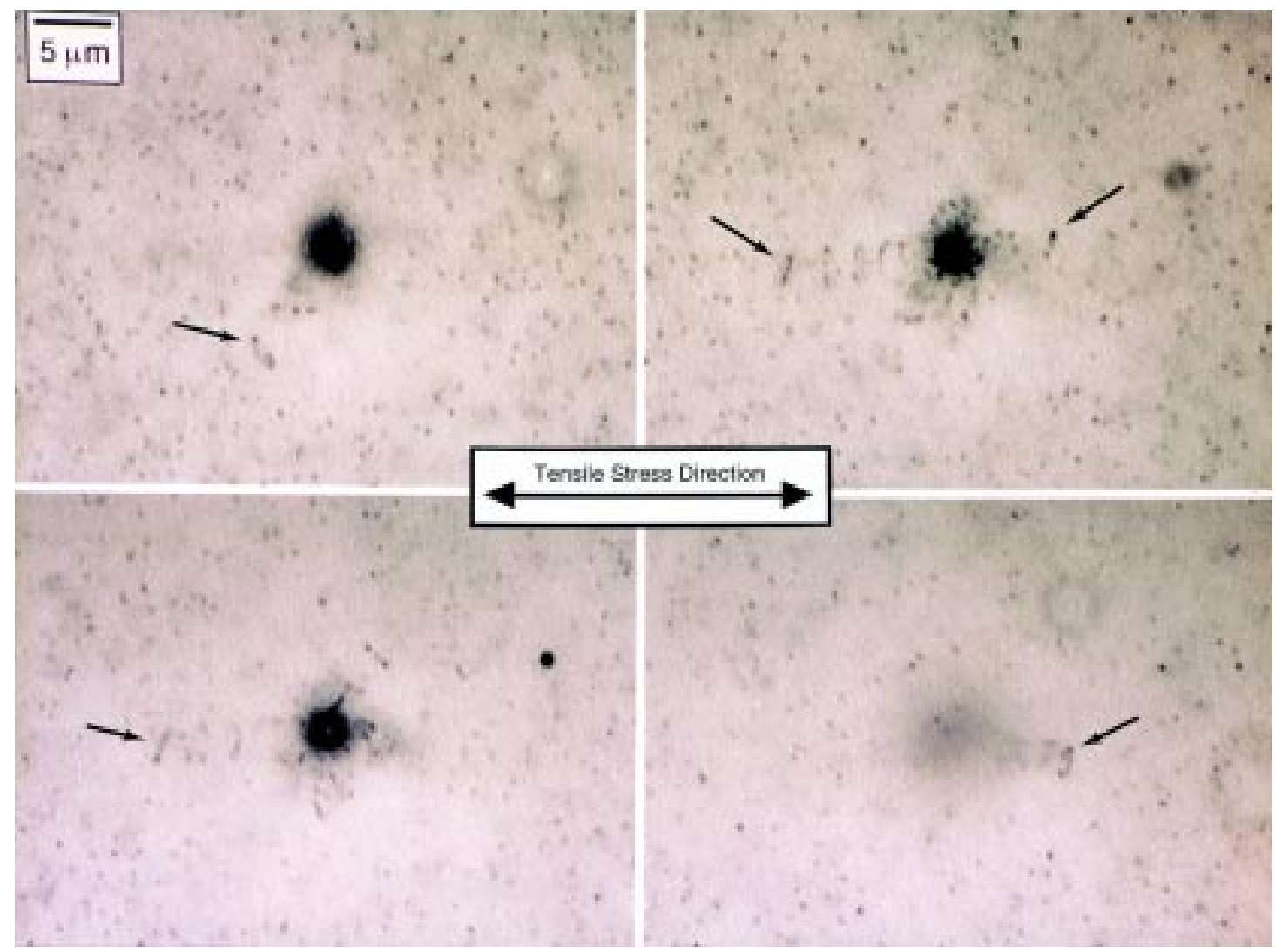

Fig. 9. Dislocation loops canting under an applied stress. This figure shows dislocations around a single glass sphere at four depths: approximately $11 \mu \mathrm{m}$ below the surface (top left), $14 \mu \mathrm{m}$ (top right), $16 \mu \mathrm{m}$ (bottom left), $23 \mu \mathrm{m}$ (bottom right). Canted isolated dislocation loops visible in each frame are indicated with an arrow. Loading direction is left-right; the far field stress was approximately $2.3 \mathrm{MPa}$.

that the applied stress direction was coincident with the data's apparent axis of symmetry.

Results from the corrected canting angle measurements on 61 loops are given in Fig. 10. For clarity, error bars are not shown in the data; estimates of experimental error are detailed in Table 1.

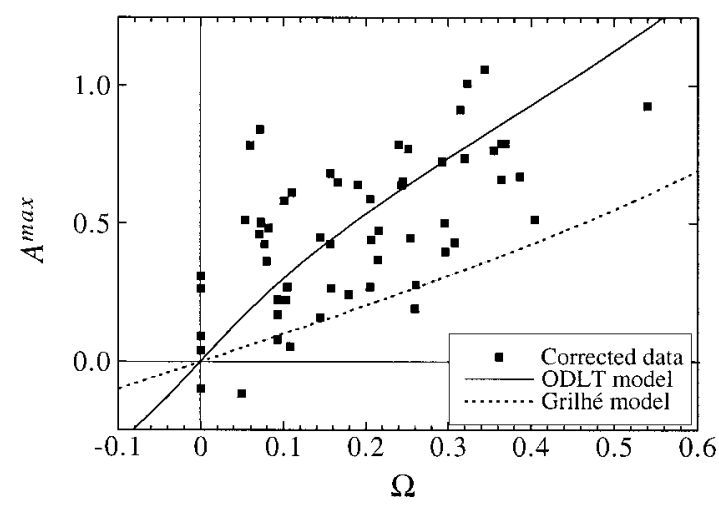

Fig. 10. Real (corrected) values of $A^{\text {max }}$ determined from a sample loaded to an applied strain of $2.3 \mathrm{MPa}$. Each data point represents the canting magnitude of a single prismatic loop. Errors associated with the measurements are discussed in the text.
The data show considerable scatter. There are two additional sources of loop canting not accommodated by the ODLT model: the previously mentioned crystal anisotropy and unrelaxed misfit stresses from the thermal quenching process. In a material with no nucleation barrier and zero friction stress, dislocation loops emitted from a sphere would glide away until the misfit stress became completely relaxed. In practice, loops become pinned during glide before complete relaxation, resulting in canted dislocation loops even under zero applied stress.

Such loop pinning should not have a preferred orientation; to confirm this, we measured the values of $A^{\text {max }}$ on 12 loops from a second sample to which no external strain was applied. The mean value obtained, $\bar{A}^{\max }$, was $0.0 \pm 0.2$; as expected, $\bar{A}^{\max }$ is near zero. The standard deviation of this measure-

Table 1. Errors associated with measurements

\begin{tabular}{lc}
\hline Loop radius, $r$ & $\pm 7 \%$ \\
Applied stress, $\sigma_{z z}$ & $\pm 0.1 \mathrm{MPa}$ \\
Friction stress, $\tau_{\mathrm{f}}$ & $\pm 0.06 \mathrm{MPa}$ \\
Measurement of canting angle & $\pm 0.03 \mathrm{rad}$ \\
Overall average relative error in $\Omega$ & $25 \%$ \\
Overall average relative error in $A^{\max }$ & $16 \%$
\end{tabular}




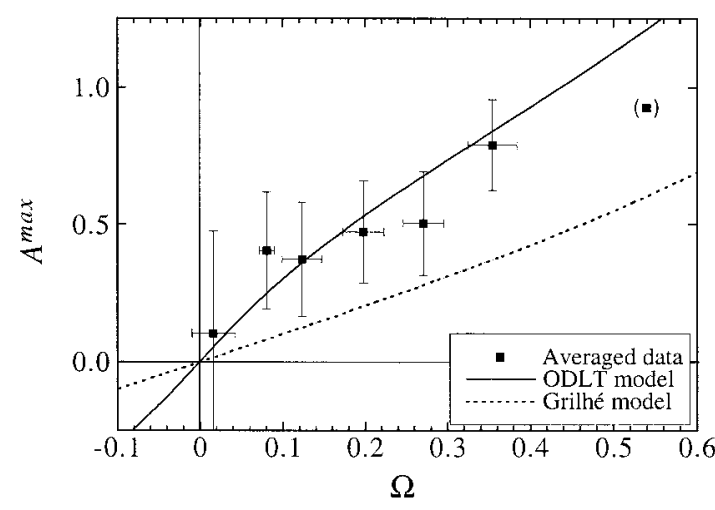

Fig. 11. Mean values of data from Fig. 10 averaged by binning the data into six groups of ten points. Error bars represent the standard deviations of the data in each bin.

ment is quite high, which suggests that the effects of unrelaxed quenching stresses are significant. As with the effects of crystal anisotropy, the mean elongation of dislocation loops should approach the predictions of the ODLT model.

We averaged the data in Fig. 10 by binning the 61 points into six bins of ten points each, the suggested minimum number of discrete observations one should consider before assuming normally distributed data [18]. The mean values of $\Omega$ and $A^{\max }$ obtained are given in Fig. 11; the averaged data are in good agreement with the ODLT model.

We have no data on loops for which $\Omega \longrightarrow 1$ because other dislocation sources activated first [11] and kept the stress low; however, it is worth speculating on the fate of supercritical $(\Omega>1)$ loops, because it has been proposed that such loops might represent a significant source of slip in crystals.

Grilhé argued that when $\Omega$ exceeds 1 , loops and helices can become Frank-Read sources. It is difficult to see how this could occur in a perfect crystal, as a Frank-Read source requires a dislocation segment to be pinned at both ends. Once the screw segments of a supercritical loop begin to cross slip, the $\hat{e}_{3}^{\text {loop }}$ components of the restoring force decrease continuously for $0 \leq v \leq 1 / 2$. The cross-slipped segments of supercritical loops should then expand across a perfect crystal while the two remnant half loops of material remain constrained in the original glide cylinder. In a real material, these mobile dislocation segments may of course become pinned or multiply via the same mechanisms which apply to ordinary glissile dislocations; however, we see no intrinsic mechanism whereby such a single expanded loop would lead to the generation of many dislocations.

\section{CONCLUSION}

We present observations and quantitative measurements of interstitial dislocation loops within bulk $\mathrm{AgCl}$ crystals subjected to externally applied stress. Data are compared with predictions for loop shape in isotropic crystals, with and without account of the orientation dependence of the dislocation line energy. The averaged loop elongation data obtained show good agreement with the orientation dependent model.

Both analyses predict that prismatic loops can cross slip and leave their glide cylinder when the value of the dimensionless stress parameter $\Omega$ reaches 1 . In terms of resolved shear stresses, the critical stress for cross slip is approximately $\tau^{*}=G b / r$.

Acknowledgements - We gratefully acknowledge support of this work from the National Science Foundation through awards DMR-9002558 and DMR-952775 under the monitorship of Bruce MacDonald. David C. Dunand of Northwestern University and Elizabeth Nugent are thanked for many helpful discussions during the course of this work.

\section{REFERENCES}

1. Nabarro, F. R. N., Theory of Crystal Dislocations. Dover, New York, 1987, pp. 374-377.

2. Read, W. T., Dislocations in Crystals. McGraw-Hill, New York, 1953, pp. 50-52.

3. Grilhé, J., Acta metall., 1963, 11, 57.

4. Ashby, M. F., in Physics of Strength and Plasticity, ed. A. S. Argon. MIT Press, Cambridge, MA, 1969, p. 113.

5. Hirth, J. P. and Lothe, J., Theory of Dislocations, reprinted 2nd edn. Krieger, Malabar, FL, 1992, pp. 1624, 157-181, 423-463.

6. Peach, M. and Koehler, J. S., Phys. Rev., 1950, 80, 436.

7. Foreman, A. J. E., Acta metall., 1955, 3, 322.

8. deWit, G. and Koehler, J. S., Phys. Rev., 1959, 116, 1113.

9. Loje, K. F. and Schuele, D. E., J. Phys. Chem. Solids, 1970, 31, 2051.

10. Bullough, R. and Newman, R. C., Phil. Mag., 1960, 5, 921.

11. Calhoun, R. B., Microplastic deformation of polycrystalline silver chloride containing a small volume fraction of hard inclusions. Ph.D. thesis, Massachusetts Institute of Technology, 1998.

12. Hedges, J. M. and Mitchell, J. W., Phil. Mag., 1953, 44, 223.

13. Mitchell, J. W., Growth and Perfection of Crystals. Wiley, New York, 1958, p. 386.

14. Jones, D. A. and Mitchell, J. W., Phil. Mag., 1958, 3, 1.

15. Mitchell, J. W., Proc. R. Soc. Lond., 1980, A371, 149.

16. Fouchaux, R. D. and Simmons, R. O., Phys. Rev. 1964, 136, A1664.

17. Dunand, D. C. and Mortensen, A., Scripta metall. mater., 1991, 25, 607.

18. Bevington, P. R. and Robinson, D. K., Data Reduction and Error Analysis for the Physical Sciences, 2nd edn. McGraw-Hill, New York, 1992, pp. 109-110.

\section{APPENDIX A}

Determining the crystallographic orientation of each grain

The orientation of each loop's glide cylinder is determined from the crystallographic orientation of each grain in the polycrystal. The grain orientations are determined 


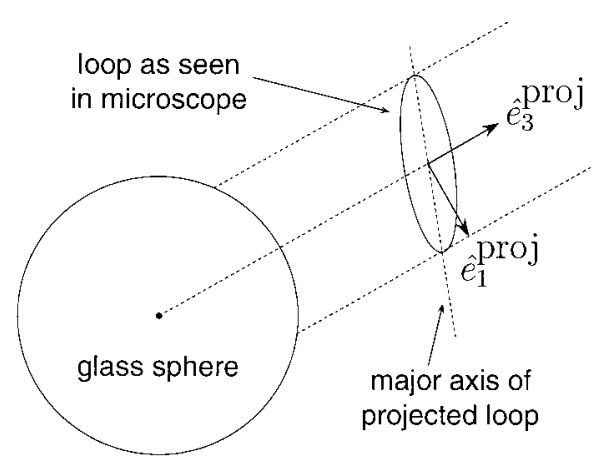

Fig. A1. Construction of coordinate system $\hat{e}_{i}^{\text {proj }}$ on microscopic images. Vector $\hat{e}_{2}^{\text {proj }}$ (not shown) is into the paper.

by measuring the angle between the loading direction and the projection of each $\langle 110\rangle$ direction onto the plane normal to the microscope axis. The grain orientation is then determined by mapping each projected angle to a particular $\langle 110\rangle$ direction and numerically calculating an orientation for this set of $\langle 110\rangle$ directions which best fits the experimental data.

The degree of fit is quantified by averaging the dot products of the normalized $\langle 110\rangle$ projections obtained from experiment with those computed. The mapping of each punching direction to a particular $\langle 110\rangle$ is done by trial and error; we assume a correct mapping has been found when the average of the normalized dot products is 0.9999 or better and there are no discrepancies between the predicted punching directions and those observed in the microscope.

\section{Compensating for the observer's frame of reference}

The $\mathrm{AgCl}$ casting technique developed by Mitchell and co-workers resulted in a strong crystallographic texture in which the microscope axis was usually very close to $\langle 100\rangle$. The "roll and recrystallize" technique used in the present work results in a more random texture. As a result, prismatic loops were rarely found in the special orientation required for the magnitude of canting to be measured directly. We used the following method to compute the actual magnitude of canting from an experimentally measurable quantity.

First, an experimentally convenient basis for making measurements, $\hat{e}_{i}^{\text {roj }}$, is defined for each loop, Fig. 1:

$$
\begin{aligned}
& \hat{e}_{1}^{\text {proj }}=k\left[\begin{array}{c}
\sin \omega \\
0 \\
-\cos \phi \cos \omega
\end{array}\right], \\
& \hat{e}_{2}^{\text {proj }}=\left[\begin{array}{l}
0 \\
1 \\
0
\end{array}\right], \\
& \hat{e}_{3}^{\text {proj }}=k\left[\begin{array}{c}
\cos \phi \cos \omega \\
0 \\
\sin \omega
\end{array}\right] .
\end{aligned}
$$

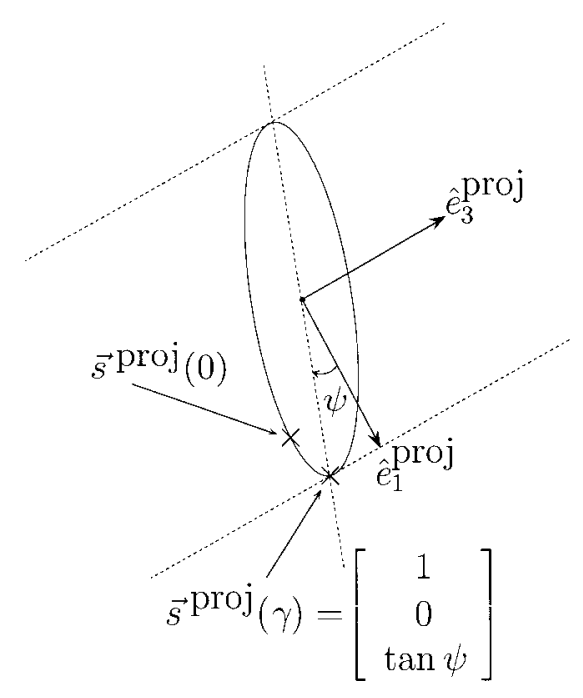

Fig. A2. Measurement of the apparent angle of canting and typical location of actual loop extrema in projection.

Here, $k=1 / \sqrt{ }\left(\cos ^{2} \phi \cos ^{2} \omega+\sin ^{2} \omega\right)$. Vector $\hat{e}_{3}^{\text {proj }}$ is the projection of the loop's Burgers vector onto the focal plane of the microscope. On a micrograph, $\hat{e}_{3}^{\text {proj }}$ is constructed by drawing a line from the center of the glass sphere through the center of the loop; $\hat{e}_{1}^{\text {proj }}$ is the normal to $\hat{e}_{3}^{\text {proj }}$ at the center of the loop (Fig. A1).

Next, a line is drawn through the major axis of the loop as it appears in the micrograph. The angle $\psi$ between the loop's major axis and $\hat{e}_{1}^{\text {proj }}$ (Fig. A2) is the apparent canting angle. We compute the real magnitude of canting numerically by finding a value of $A^{\max }$ which would result in the apparent angle of canting observed.

To do this, we write the equation describing the loop in the $\hat{e}_{i}^{\text {proj }}$ frame, yielding $\vec{s}^{\text {proj }}(\theta)=M \cdot \vec{s}(\theta)$ where $M_{i j}=\hat{e}_{i}^{\text {proj }} \cdot \hat{e}_{j}^{\text {loop }}$. For $\alpha=0$, the maximum elongation of the loop occurs at $\theta=0$ in the $\hat{e}_{i}^{\text {loop }}$ frame. In micrographs, the location of the real maximum $\vec{s}^{\text {proj }}(0)$ will not generally coincide with the apparent maximum; rather, it will be found at some other value of $\theta$ which we call $\gamma$ (Fig. A2). To compute $\gamma$, we find the value of $\theta$ for which $s_{1}^{\text {proj }}(\theta)=1$, i.e. where the projected loop touches the projection of its glide cylinder. This is a function of $\omega$ and $\phi$ only.

Once $\gamma$ is known, we find a value of $A^{\max }$ such that $s_{3}^{\text {proj }}(\gamma)=\tan \psi$. Here we assume that loops elongate by canting around the $\hat{e}_{2}^{\text {loop }}$ axis as predicted by the model; this should be true on average.

The robustness of the algorithm was checked by numerically generating loops of random elongation and orientation and comparing the elongation of each generated loop with the value back-calculated using the method above. The algorithm always worked with synthetic data. With real data, some obviously incorrect results were generated, especially when the loops were at a high angle above or below the microscope's focal plane. These points were discarded. 